\title{
Inhibition of inflammation by SP600125 in cholestatic liver injury is dependent on the administration-based exposure profile
}

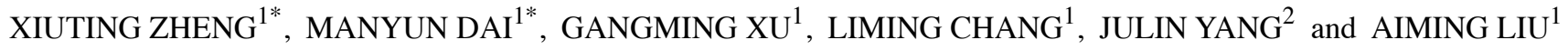 \\ ${ }^{1}$ Department of Pharmacology, Medical School of Ningbo University, Ningbo, Zhejiang 315211; \\ ${ }^{2}$ Department of Basic Nutrition, Ningbo College of Health Sciences, Ningbo, Zhejiang 315100, P.R. China
}

Received November 16, 2019; Accepted September 11, 2020

DOI: $10.3892 /$ ijmm.2020.4742

\begin{abstract}
SP600125 is a classic inhibitor of c-Jun-N-terminal kinase $(\mathrm{JNK})$ that is widely used in numerous medicinal studies, but its administration regimen has yet to be optimized. In the present study, intraperitoneal (i.p.) and intragastric (i.g.) injections of $15 \mathrm{mg} / \mathrm{kg}$ SP600125 was performed in mice to compare the inhibitory effect against JNK signalling in cholestasis induced by $\alpha$-naphthylisothiocyanate (ANIT). SP600125 at a dose of $15 \mathrm{mg} / \mathrm{kg}$ administered by i.p. substantially decreased ANIT-induced liver injury as observed by biochemical and histopathological examinations. The adaptation of bile acid synthesis was inhibited in the A-SP-i.p. group compared with that in the A-SP-i.g. group, as indicated by the expression analysis of CYP7A1 and CYP8B1. The transcription of the pro-inflammatory factors $I L-6, I L-1 \beta$, $I C A M-1$ and $I L-10$ supported the differential toxic responses. Western blot analysis revealed that JNK signalling activated by ANIT was inhibited more markedly in the A-SP-i.p. group than in the A-SP-i.g. group. The peak concentration and the $\mathrm{AUC}_{0-24}$ of SP600125 in the A-SP-i.p. group were 5-fold and 1.56-fold higher, respectively, compared with those in the A-SP-i.g. group. These data indicated that i.p. administration of SP600125 produced a high plasma exposure profile, which directly determined its efficacy of blocking the JNK signalling. This effect of SP600125 on the JNK pathway may provide an optimized design for future in vivo investigations.
\end{abstract}

\section{Introduction}

c-Jun-N-terminal kinase (JNK) is a member of the mitogen-activated protein kinase (MAPK) family and includes

Correspondence to: Professor Aiming Liu, Department of Pharmacology, Medical School of Ningbo University, 818 Fenghua Road, Ningbo, Zhejiang 315211, P.R. China

E-mail: liuaiming@nbu.edu.cn

*Contributed equally

Key words: JNK, SP600125, administration route, inflammation, cholestasis
3 isoforms in mammals, namely JNK1, JNK2 and JNK3 (1). JNK1 and JNK2 are expressed in almost all cells, whereas JNK3 is mainly expressed in the brain, heart, and testis (2). The JNK pathway may be activated by cytokines, pathogens, toxins, drugs and metabolic changes (3). JNK activation is involved in cell death, differentiation, proliferation, and tumorigenesis in hepatocytes (4). In hepatic macrophages and hepatic stellate cells, JNK was revealed to contribute to liver inflammation and fibrosis (5). The JNK pathway also regulates inflammation, and insulin resistance, which are associated with hepatic diseases such as non-alcoholic steatosis, fibrosis, and hepatocellular carcinoma (3).

SP600125 is widely used in biochemical studies as a selective JNK inhibitor (6) and it can block the activation of JNK to modulate the expression of inflammatory factors (6-8). Additionally, this inhibitor has been used in numerous cellular models to investigate the underlying pathophysiological and pharmacological mechanisms. Incubation with bile acid significantly reduced L02 cell viability, and this effect was reduced by pre-treatment with $20 \mu \mathrm{M}$ SP600125, which involved downregulation of $I L-1 \beta$ and inhibition of JNK phosphorylation (9). In MC3T3-E1 cells, 25 and $50 \mu \mathrm{M}$ SP600125 reduced osteoblast apoptosis in an LPS-induced model and restored differentiation of osteoblasts (10).

In a rat model, administration of $30 \mathrm{mg} / \mathrm{kg} \mathrm{SP} 600125$ by intraperitoneal (i.p.) injection once per day for 8 weeks suppressed autophagy and attenuated insulin resistance by inhibiting JNK signalling (11). In C57BL/6 mice, a single dose of 10 and $30 \mathrm{mg} / \mathrm{kg}$ SP600125 by i.p. injection inhibited 3-chloro-1,2-propanediol esters-induced apoptosis by downregulating the $\mathrm{JNK} / \mathrm{p} 53$ pathway in tubular cells (12). In a type 1 diabetic mouse model induced by streptozocin, $5 \mathrm{mg} / \mathrm{kg}$ SP600125 by intragastric (i.g.) injection every other day for 3 months inhibited cardiac fibrosis, oxidative stress, endoplasmic reticulum stress, and cell death by inhibiting the JNK pathway $(13,14)$. However, the optimal administration regimen of SP600125 inhibiting the JNK pathway and its exposure profile have yet to be determined.

Cholestasis is caused by disrupted structure and impaired function of the hepatobiliary system, which occurs in a number of clinical disorders (15). Patients with cholestasis may eventually develop sepsis, immune depression, cardiovascular, hepatic and renal failure (16). Cholestatic liver injury is closely associated with several inflammatory pathways $(17,18)$. It was 
reported that the synthesis and excretion of bile acids increased the levels of TNF- $\alpha$ and IL-1 $\beta$ in hepatic Kupffer cells (19). In addition, cytokines, cholic acid and deoxycholic acid have been revealed to activate the JNK/c-Jun pathway $(20,21)$. In cellular models, the JNK pathway was also found to regulate bile acid metabolism $(22,23)$. Thus, $\alpha$-naphthylisothiocyanate (ANIT)-induced cholestatsis is a suitable model for investigating the inhibitory effect of SP600125 on the JNK pathway.

In the present study, ANIT-induced cholestatic liver injury was used to evaluate the inhibitory effect of SP600125 administered by i.p. and i.g. injections, on the JNK pathway. The data were revealed to be informative in designing biomedical experiments where SP600125 may be used.

\section{Materials and methods}

Chemicals and reagents. ANIT was purchased from Sigma-Aldrich; Merck KGaA. Alkaline phosphatase (ALP), total bile acid (TBA), alanine aminotransferase (ALT), and aspartate aminotransferase (AST) assay kits were purchased from Ningbo Ruiyuan Biotechnology Co., Ltd. TRIzol ${ }^{\circledR}$ and the reverse transcription kit were purchased from Invitrogen (Thermo Fisher Scientific, Inc). The LightCycle 480 SYBR Green I Master Mix was obtained from Roche Diagnostics. Antibodies against phosphorylated (p)-MAPK kinase 4 (MKK4) (1:1,000; product no. 4514) and total (t)-MKK4 (1:1,000; product no. 9152), t-JNK (1:1,000; product no. 9252) and p-JNK (1:1,000; product no. 9912), p-c-Jun $(1: 1,000$; product no. 2361) and t-c-Jun (1:1,000; product no. 9165), were obtained from Cell Signalling Technology, Inc. Primary antibodies against CYP7A1 (1:1,000; product code ab65596), CYP8B1(1:1,000; product code ab191910), GAPDH (1:10,000; product code ab181602) were acquired from Abcam. Secondary antibody IgG H\&L (HRP) was also obtained from Abcam (1:2,000; product code ab205718). All the other chemicals were of the highest grade available from commercial sources.

Animals and treatment. A total of 50 mice were maintained under a 12-h light/dark cycle with free access to water and a commercial diet. The SPF room housing the mouse cages was set at $23 \pm 1^{\circ} \mathrm{C}$ with a relative humidity of $60-70 \%$. All the mice were allowed to acclimate for 7 days before the experiments. The procedures performed were approved by the Animal Care and Use Committee of Ningbo University. Twenty of the mice were assigned into 4 groups ( $n=5 /$ group) as follows: Negative control (Con), ANIT/positive control (ANIT), ANIT/SP600125-i.p. (A-SP-i.p.) and ANIT/SP600125-i.g. (A-SP-i.g.). A single dose of $15 \mathrm{mg} / \mathrm{kg} \mathrm{SP600125} \mathrm{dissolved} \mathrm{in}$ corn oil was administered by i.p. or i.g. injections to pre-treat the mice. At 30 min after SP600125 administration, a single dose of $75 \mathrm{mg} / \mathrm{kg}$ ANIT in corn oil was injected to induce cholestasis. At $48 \mathrm{~h}$ after the treatment, the mice were weighed and then killed by asphyxiation after blood collection. To perform euthanasia, the mouse cage was moved into a transparent polycarbonate chamber. Compressed $\mathrm{CO}_{2}$ gas (purity $>99 \%$ ) was introduced at a rate of $30 \%$ chamber volume per min using a $\mathrm{CO}_{2}$-specific regulator. Death was confirmed by observing absence of respiratory movement and faded eye colour. The chamber was cleaned thoroughly to remove any potential risk for euthanasia. Liver tissues and the gallbladder were harvested for calculation of liver and gallbladder indices. Half of the isolated liver tissues were cut and fixed in $10 \%$ neutral buffered formalin at room temperature for $24 \mathrm{~h}$. The remaining liver tissues were immediately frozen and stored under at $-80^{\circ} \mathrm{C}$.

Biochemical analysis and histopathological assessment. The Multiskan GO (Thermo Fisher Scientific, Inc.) was used to measure the TBA, ALP, ALT and AST levels in the serum according to the manufacturer's instructions.

The liver tissues were dehydrated through serial concentrations of alcohol $(70,80,90$ and $100 \%)$ and xylene, followed by paraffin embedding at $60 \mathrm{C}$ and cooled to $-20 \mathrm{C}$. The paraffin block was cut into $4-\mu \mathrm{m}$ sections which were then stained with hematoxylin for $3 \mathrm{~min}$ and eosin for $2 \mathrm{~min}$ at room temperature. A BX51 light microscope (Olympus Corporation) at x40 and $\mathrm{x} 400$ magnification and ten serial sections per preparation were used to randomly capture histopathological images.

Quantitative PCR (qPCR) and western blot (WB) analysis. Processing of liver samples for the qPCR and WB analysis followed previously published procedures with slight modifications (18). Specifically, a 5- $\mu 1$ PCR system was designed for the 384 plate. For qPCR analysis, the primers (Table SI) were also obtained from a public database (http://mouseprimerdepot.nci.nih.gov). This system configuration followed the protocols provided by the manufacturer. Melting curves were used to assess primer specificity. 18S rRNA was used as an internal standard to calculate the relative transcription level for each run

Concentration-time profile of SP600125. In a second pilot experiment, 30 mice were divided into 6 groups ( $n=5 /$ group), as follows: SP600125-i.p.-A (SP-i.p.-A), SP600125-i.p.-B (SP-i.p.-B), SP600125-i.p.-C (SP-i.p.-C), SP600125-i.g.-A (SP-i.g.-A) SP600125-i.g.-B (SP-i.g.-B) and SP600125-i.g.-C (SP-i.g.-C). A single dose of $15 \mathrm{mg} / \mathrm{kg}$ SP600125 was administered by i.p. and i.g. injections. Blood (30 $\mu \mathrm{l})$ was collected from the tail vein for exposure assessment. The blood collection was performed at 0.3, 1.5, 3, 6 and $14 \mathrm{~h}$ following SP600125 treatment in the SP-i.p.-A and SP-i.g.-A groups. Similarly, the blood collection in the SP-i.p.-B and SP-i.g.-B groups was performed at 0.5, 2, 8 and 18 h. For the SP-i.p.-C and SP-i.g.-C groups, blood was collected at 1, 4, 10 and $24 \mathrm{~h}$ after SP600125 treatment. The determination followed previously published procedures (18).

Statistical analysis. All data were expressed as the mean \pm SD. GraphPad Prism (version 7.0; GraphPad Software, Inc.) for Windows was used for the data analysis. Two-samples t-test was used to test the difference between two groups, and Bonferroni's correction was performed for all P-values. The $\mathrm{C}_{\max }$ and AUC were calculated using Drug and Statistics (Version 3.0; Mathematical Pharmacology Professional Committee of China, Shanghai, China) (24).

\section{Results}

Cholestatic liver injury is differentially inhibited. The levels of the biochemical markers ALP, TBA, ALT and AST in 

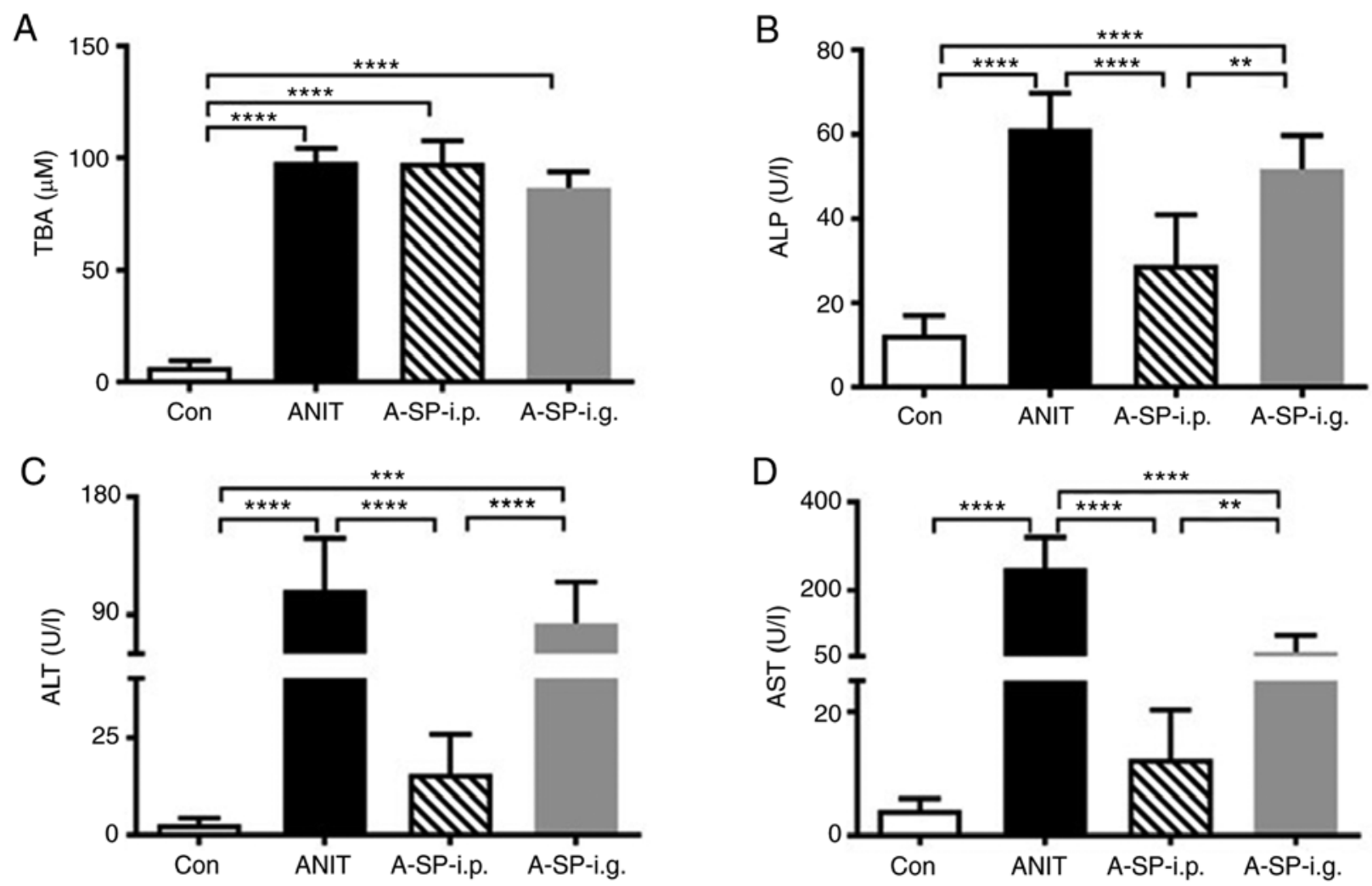

Figure 1. Biochemical markers indicating the inhibitive effect of SP600125 against intrahepatic cholestasis. (A) TBA, (B) ALP, (C) ALT and (D) AST in the mouse serum of four groups $48 \mathrm{~h}$ after ANIT administration. The data are expressed as the mean $\pm \mathrm{SD}$. (n=5). ${ }^{* *} \mathrm{P}<0.01,{ }^{* * *} \mathrm{P}<0.001$ and ${ }^{* * * *} \mathrm{P}<0.0001$. TBA, total bile acid; ALP, alkaline phosphatase; ALT, alanine aminotransferase; AST, aspartate aminotransferase; ANIT, $\alpha$-naphthylisothiocyanate; A-SP-i.p., ANIT/SP600125-i.p.; A-SP-i.g., ANIT/SP600125-i.g.; i.p, intraperitoneal; i.g., intragastric; Con, negative control.

the serum were all markedly increased in the ANIT group compared with those in the control group (Fig. 1 ; all $\mathrm{P}<0.0001$ ). The serum TBA levels in the A-SP-i.p. and A-SP-i.g. groups were similar to those in the ANIT group (Fig. 1A). In the A-SP-i.p. group, but not in the A-SP-i.g. group, the ALP, ALT and AST levels in serum were decreased compared with those in the ANIT group (Fig. 1B-D; all P<0.0001). The hepatic pathological analysis in the control group revealed characteristic features (Fig. 2A). Loss of cellular boundaries, degenerative changes and marked necrosis were identified in the ANIT group (Fig. 2B). The A-SP-i.p. group, but not the A-SP-i.g. group, exhibited no significant difference compared with the control group (Fig. 2C and D). Thus, pre-treatment with SP600125 at a dose of $15 \mathrm{mg} / \mathrm{kg}$ by i.p. injection reversed the liver injury induced by ANIT in mice, although the serum TBA level did not improve. However, the aforementioned protective effect was not observed in the mice pre-treated with SP600125 at $15 \mathrm{mg} / \mathrm{kg}$ by i.g. injection.

Adaptation of bile acid metabolism and transport. The rate-limiting enzymes CYP7A1 and CYP8B1 play an important role in bile acid homeostasis (25). ANIT challenge decreased the $C Y P 7 A 1$ and $C Y P 8 B 1$ mRNA levels in the ANIT group compared with those in the control group (Fig. $3 \mathrm{~A}$ and $\mathrm{B}$; $\mathrm{P}<0.01$ and $\mathrm{P}<0.001)$. In the A-SP-i.p. group, but not in the A-SP-i.g. group, both $C Y P 7 A 1$ and $C Y P 8 B 1$ mRNA levels were increased compared with those in the ANIT group (Fig. $3 \mathrm{~A}$ and $\mathrm{B} ; \mathrm{P}<0.05$ and $\mathrm{P}<0.01$ ). In the $\mathrm{A}-\mathrm{SP}-$ i.g. group, CYP7A1 and CYP8B1 mRNA levels were lower compared
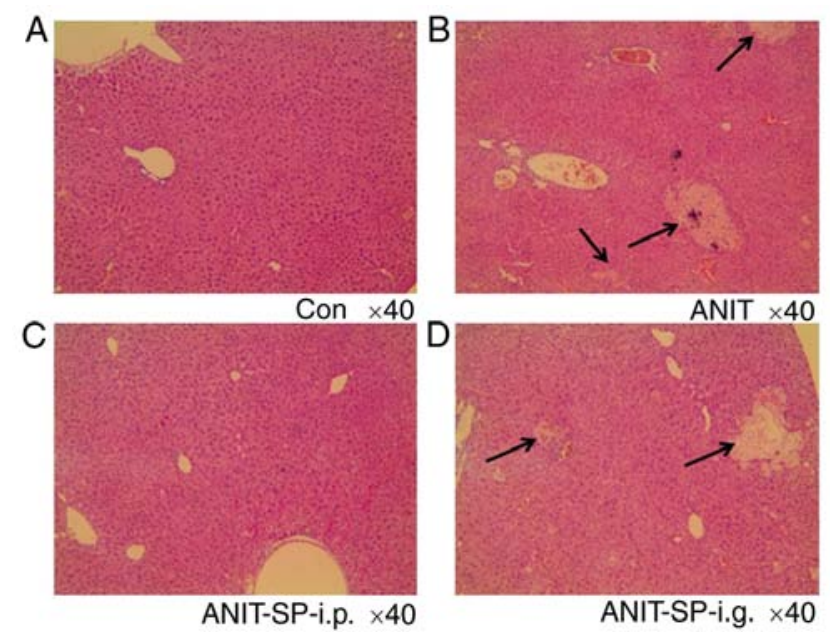

Figure 2. Histopathology of liver tissues indicating the inhibitive effect of SP600125 against hepatotoxicity induced by ANIT treatment. (A) H\&E staining of liver tissues in the Con group. (B) H\&E staining of liver tissues in the ANIT grou $p$ with significant necrosis. (C) H\&E staining of liver tissues in the A-SP-i.p. group where no significant necrosis was observed. (D) H\&E staining of liver tissues in the A-SP-i.g. group with significant necrosis. Arrows indicate a loss of cellular boundaries, degenerative changes and marked necrosis. ANIT, $\alpha$-naphthylisothiocyanate; H\&E, hematoxylin and eosin; Con, negative control; A-SP-i.p., ANIT/SP600125-i.p.; A-SP-i.g., ANIT/SP600125-i.g.; i.p, intraperitoneal; i.g., intragastric.

with those in the A-SP-i.p. group, as the adaptation remained strong $(\mathrm{P}<0.05$ and $\mathrm{P}<0.01)$. In the $\mathrm{WB}$ analysis, the protein levels of CYP7A1 and CYP8B1 in the A-SP-i.g. group were 

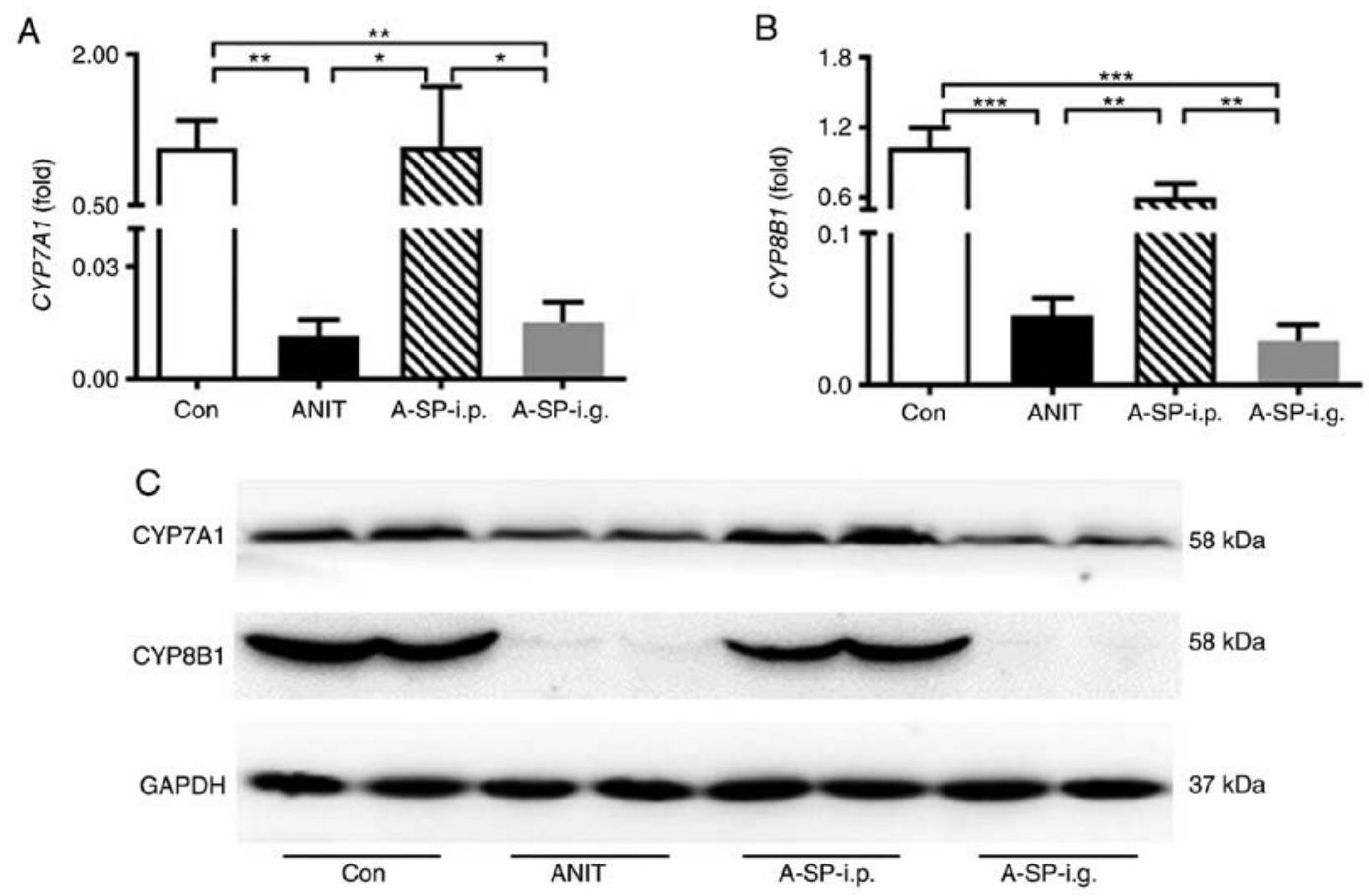

Figure 3. Expression of genes and proteins involved in bile acid synthesis revealing different adaptation caused by SP600125 treatment. (A) CYP7A1 mRNA levels in mice of four groups $48 \mathrm{~h}$ after the ANIT administration. (B) CYP8B1 mRNA in mice of four groups $48 \mathrm{~h}$ after the ANIT administration. (C) Western blot analysis of CYP7A1 and CYP8B1 levels in the four groups $48 \mathrm{~h}$ after the ANIT administration. Data were from liver samples collected $48 \mathrm{~h}$ after ANIT treatment and $2 / 5$ of the liver tissues were randomly selected for western blot analysis. The mRNA levels were measured by qPCR and normalized by $18 S$ $r R N A$. mRNA levels in the vehicle-treated control mice were set as 1 and the results are expressed as the mean $\pm \mathrm{SD}$. $(\mathrm{n}=5)$. ${ }^{*} \mathrm{P}<0.05,{ }^{* * *} \mathrm{P}<0.01$ and ${ }^{* * * *} \mathrm{P}<0.001$. ANIT, $\alpha$-naphthylisothiocyanate; Con, negative control; A-SP-i.p., ANIT/SP600125-i.p.; A-SP-i.g., ANIT/SP600125-i.g.; i.p, intraperitoneal; i.g., intragastric.
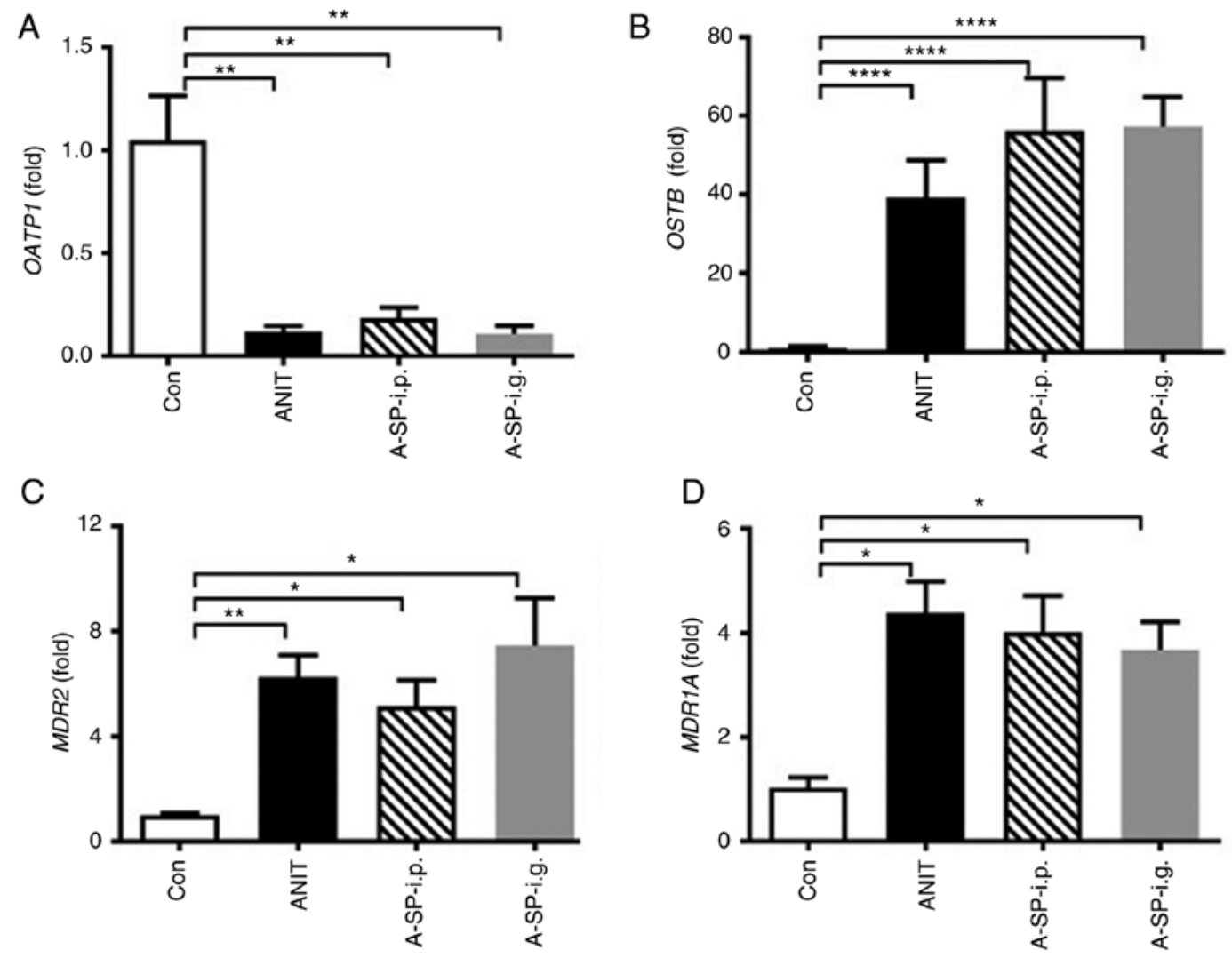

Figure 4. Adaptation of genes involved in bile acid efflux transport. (A) OATP1, (B) OSTB, (C) MDR2 and (D) MDR1A mRNA levels in four groups $48 \mathrm{~h}$ after the ANIT administration. mRNAs levels in the vehicle-treated control mice were set as 1 and the results are expressed as the mean \pm SD. (n=5). $\mathrm{P}<0.05$, ${ }^{* *} \mathrm{P}<0.01$ and ${ }^{* * * * *} \mathrm{P}<0.0001$. OATP1, organic anion-transporting polypeptide 1; OSTB, organic solute transporter $\beta$ subunit; MDR2, multidrug resistance 2; MDR1A, multidrug resistance 1A; Con, negative control; ANIT, $\alpha$-naphthylisothiocyanate; A-SP-i.p., ANIT/SP600125-i.p.; A-SP-i.g., ANIT/SP600125-i.g.; i.p, intraperitoneal; i.g., intragastric. 


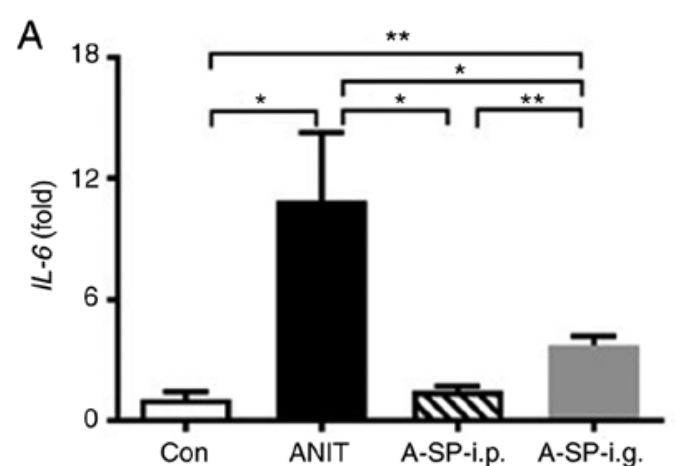

C

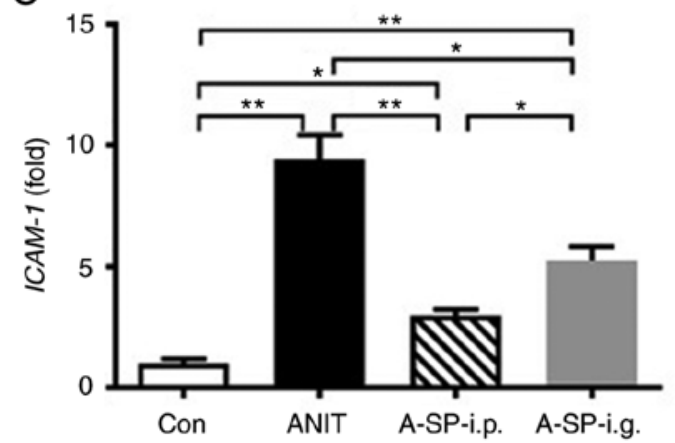

$\mathrm{E}$

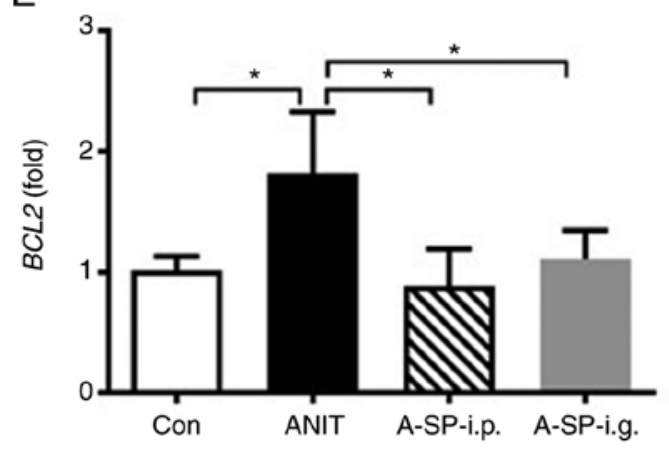

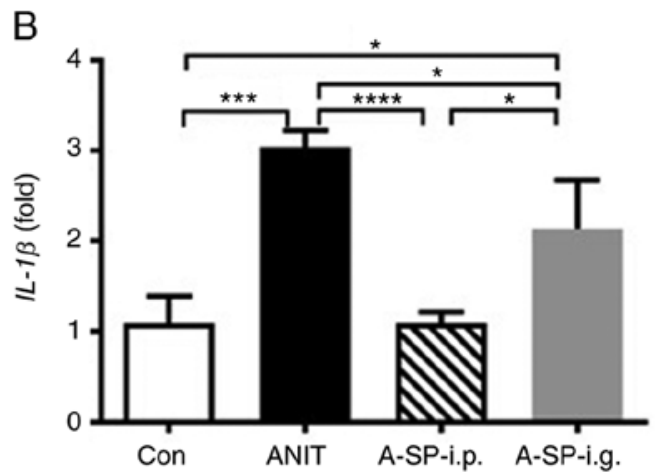

D

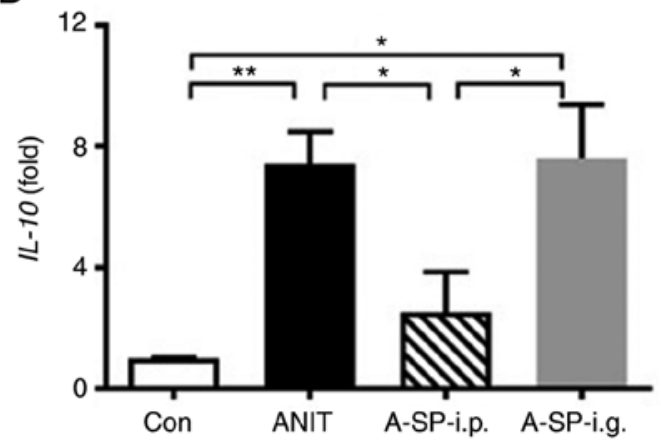

F

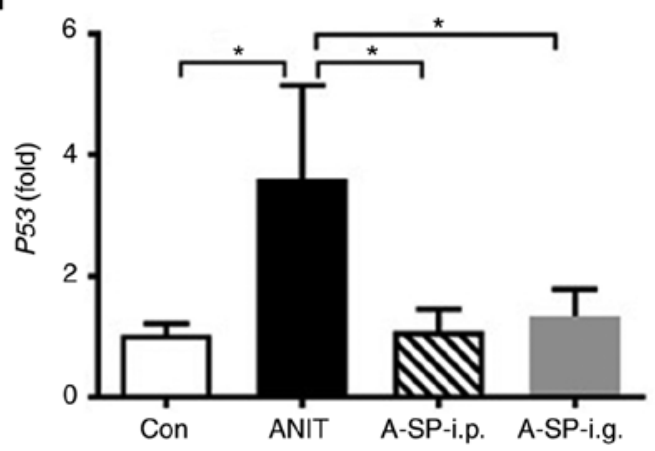

Figure 5. Levels of mRNAs encoding inflammation-related factors and apoptosis genes. (A) $I L-6$, (B) $I L-1 \beta$, (C) $I C A M-1$, (D) $I L-10$, (E) $B C L 2$ and (F) $P 53$ mRNA levels in four groups $48 \mathrm{~h}$ after the ANIT administration. The mRNA levels were measured by qPCR and normalized by 185 rRNA. mRNA levels in the vehicle-treated control mice were set as 1 and the results are expressed as the mean $\pm \mathrm{SD} . \mathrm{n}=5 .{ }^{*} \mathrm{P}<0.05,{ }^{* *} \mathrm{P}<0.01,{ }^{* * *} \mathrm{P}<0.001$ and ${ }^{* * * *} \mathrm{P}<0.0001$. Con, negative control; ANIT, $\alpha$-naphthylisothiocyanate; A-SP-i.p., ANIT/SP600125-i.p.; A-SP-i.g., ANIT/SP600125-i.g.; i.p, intraperitoneal; i.g., intragastric.

decreased compared with those in the control group, similar to those in the ANIT group. In the A-SP-i.p. group, these levels were almost identical to those in the control group (Fig. 3C).

Organic anion-transporting polypeptide 1 (OATP1), heteromeric organic solute transporter $\beta$ subunit (OSTB), multidrug resistance 2 (MDR2) and multidrug resistance $1 \mathrm{~A}$ (MDR1A) are involved in bile acid efflux (25). OATP1 was decreased, and $O S T B, M D R 2, M D R 1 A$ were all increased by ANIT treatment. However, no difference of these transporters was observed among the A-SP-i.p., A-SP-i.g. and ANIT groups. The changes in bile acid transporters were similar in the three groups challenged by ANIT, which was mainly considered to be adaptive responses (Fig. 4A-D). Other genes involved in bile acid metabolism were not obviously affected (data not shown).

Differential inflammation responses between the two administration routes. The mRNA expression levels of $I L-6$, $I L-1 \beta$ and $I C A M-1$ was revealed to be significantly increased following ANIT treatment (Fig. 5A-C, $\mathrm{P}<0.05, \mathrm{P}<0.001$ and $\mathrm{P}<0.01) . I L-6$ and $I L-1 \beta \mathrm{mRNA}$ levels were decreased in the A-SP-i.p. and A-SP-i.g. groups, but the decrease was greater in the A-SP-i.p. group compared with the ANIT group. The protective inflammation factor $I L-10$ was upregulated in the ANIT and A-SP-i.g. groups compared with the control group, which was not observed in the A-SP-i.p. group (Fig. 5D). In terms of apoptosis, the BCL2 and P53 mRNA levels were increased following administration of ANIT at $75 \mathrm{mg} / \mathrm{kg}$, but both were suppressed by pretreatment with SP600125, without a significant difference between the two administration routes (Fig. 5E and F). These data indicated that inflammation developed in the mice challenged with ANIT, but it was differentially blocked by i.p. and i.g. administration of SP600126.

SP600125 i.p. inhibits JNK signalling. The $c-J U N$ and $c$-FOS mRNA levels were increased by ANIT. Compared with the ANIT group, the $c-J U N$ mRNA was decreased in 
A

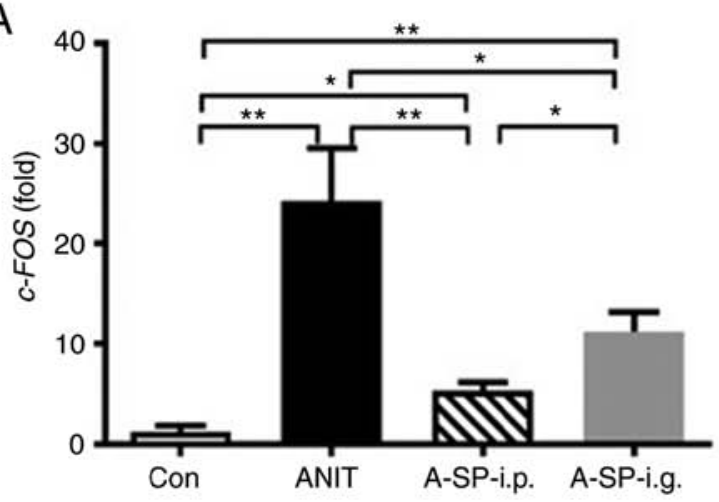

B

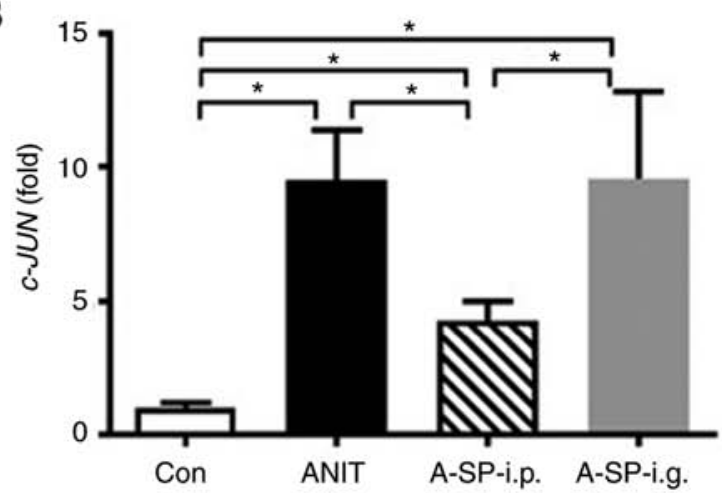

C p-MKK4

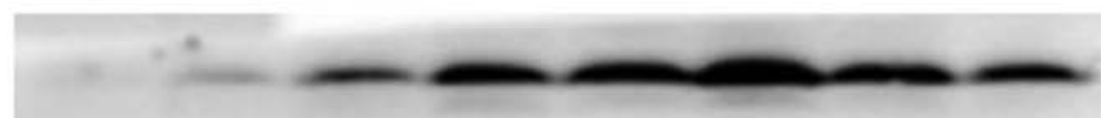

$44 \mathrm{kDa}$

t-MKK4

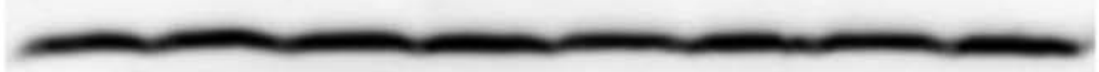

$44 \mathrm{kDa}$
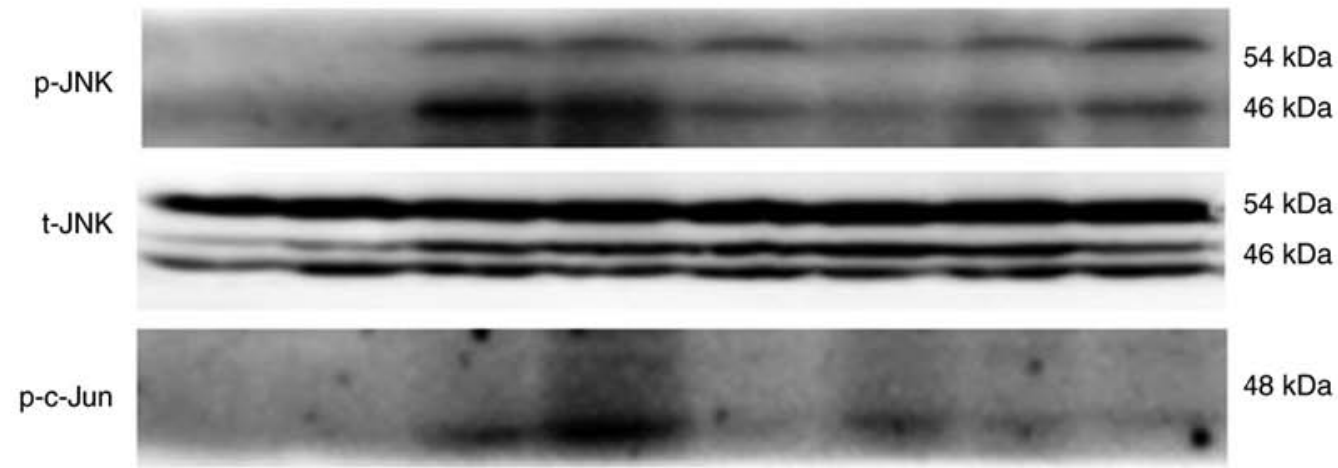

t-c-Jun

GAPDH

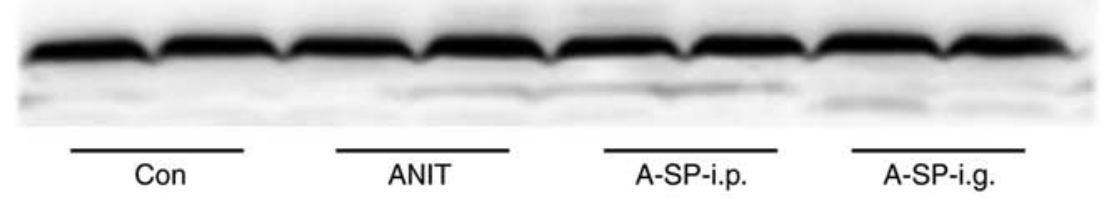

$37 \mathrm{kDa}$

Figure 6. SP600125 blocks JNK signaling in i.p. administration. (A and B) $c$-JUN, $c$-FOS mRNA levels in mice of four groups 48 h after the ANIT administration. (C) Western blot analysis of components of the JNK signaling pathway in liver extracts and data were from liver samples collected $48 \mathrm{~h}$ after ANIT treatment and $2 / 5$ of the liver tissues were randomly selected for western blot analysis. GAPDH was used as a loading control. The mRNA levels were measured by qPCR and normalized by $18 S \mathrm{SRNA}$. mRNA levels in the vehicle-treated control mice were set as 1 and the results are expressed as the mean \pm SD. $\mathrm{n}=5$. ${ }^{*} \mathrm{P}<0.05$ and ${ }^{* *} \mathrm{P}<0.01$. JNK, c-Jun-N-terminal kinase; Con, negative control; ANIT, $\alpha$-naphthylisothiocyanate; A-SP-i.p., ANIT/SP600125-i.p.; A-SP-i.g., ANIT/SP600125-i.g.; i.p, intraperitoneal; i.g., intragastric.

the A-SP-i.p. group $(\mathrm{P}<0.05)$, but not in the A-SP-i.g. group (Fig. 6A). The $c$-FOS mRNA was decreased in both the A-SP-i.p. and A-SP-i.g. groups, and this decrease was greater in the A-SP-i.p. group (Fig. 6B, $\mathrm{P}<0.01$ and $\mathrm{P}<0.05$ ). The WB analysis revealed that the p-MKK4 was activated in the ANIT, A-SP-i.p. and A-SP-i.g. groups and p-JNK was activated in the ANIT group compared with the control group. This pathway was strongly inhibited in the A-SP-i.p. group, but only partly inhibited in the A-SP-i.g. group. Activation of p-c-Jun was observed in the ANIT group, but it was markedly inhibited by SP600125 in the A-SP-i.p. group, in which liver injury was not observed (Fig. 6C). Thus, SP600125 acted against ANIT-induced cholestatic liver injury following i.p. administration. The aforementioned protective effect and differential inflammation regulation were attributed to the inhibition of JNK signalling following i.p. administration.

SP600125 exposure assessment. The multiple reaction monitoring mode was used to detect the negative ion transition $\mathrm{m} / \mathrm{z}$ 191/219 for SP600125 (Fig. 7A and B). The calibration curve 

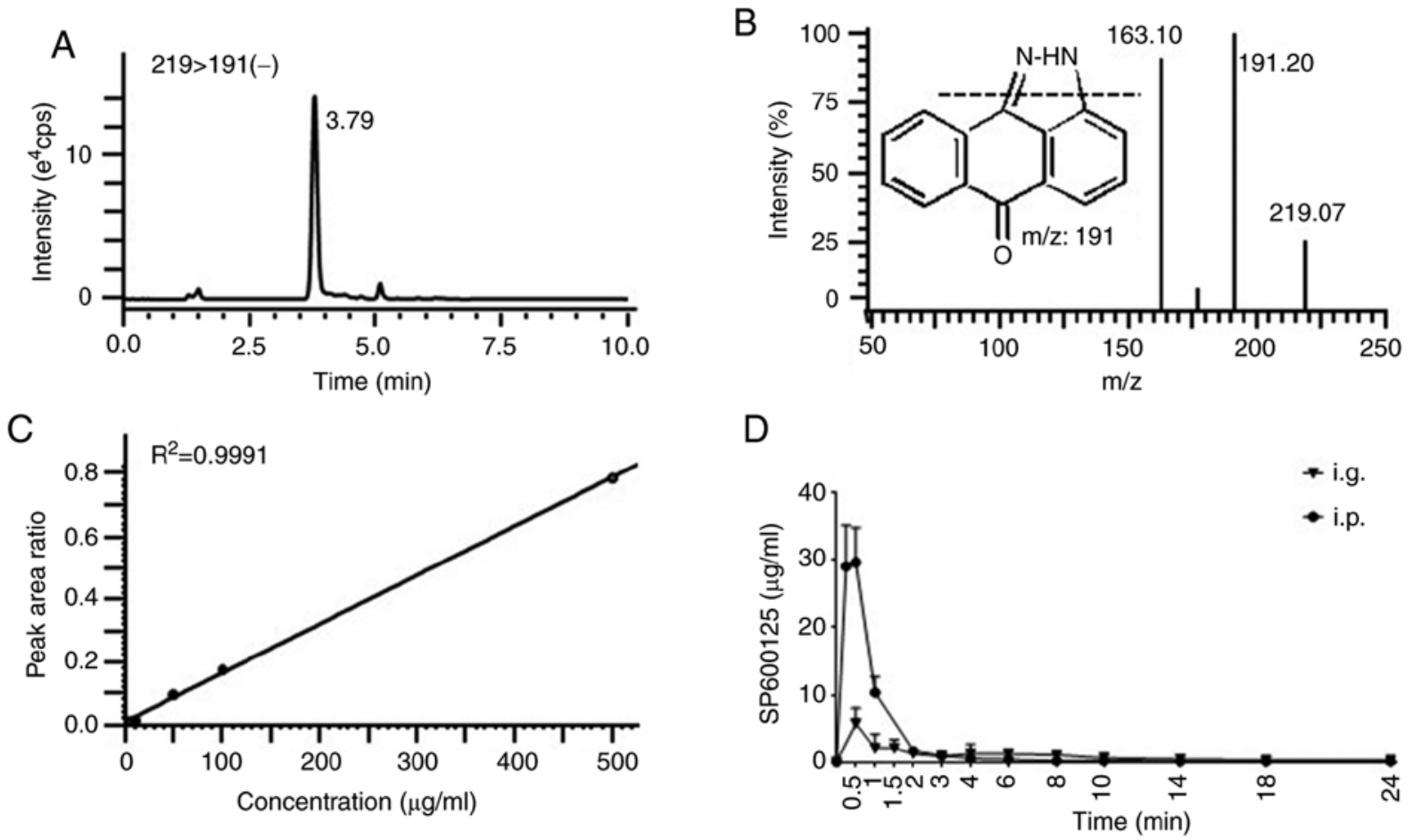

Figure 7. Different plasma concentration-time profiles of SP600125 after different administration routes. (A) Ion count of $m / z$ 219/191 of ANIT-treated mouse serum sample in negative detection mode. (B) MS/MS spectra of the peak at retention time 3.79 in mouse serum in panel A and the proposed fragmentation mode. (C) The calibration curve used for quantification of SP600125 concentration in the serum. (D) The concentration-time profiles of SP600125 in the serum following two different administration routes. ANIT, $\alpha$-naphthylisothiocyanate.

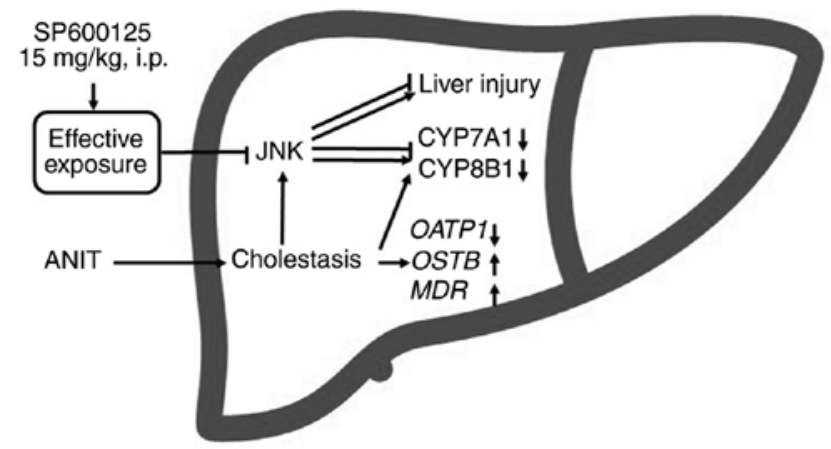

Figure 8. ANIT induces evident liver injury in addition to strong adaptation of bile acid synthesis and transport in the mice. SP600125 $15 \mathrm{mg} / \mathrm{kg}$ by i.p. produces an effective exposure level compared with i.g. administration, which inhibits the JNK pathway and then liver injury. The adaptation of synthesizing CYP7A1 and CYP8B1, not the other genes involved in bile acid metabolism is reversed by SP600125, suggesting that adaptation of two genes is dependent on the JNK pathway. This action mode of SP600125 against JNK pathway offers an optimized experimental design for in vivo investigations. ANIT, $\alpha$-naphthylisothiocyanate; i.p, intraperitoneal; i.g., intragastric.

demonstrated a good linear association $\left(\mathrm{R}^{2}>0.99\right)$ with satisfactory chromatographic performance (Fig. 7C). The mean plasma concentration-time profiles after a single administration of SP600125 (15 mg/kg i.p. and i.g.) are presented in Fig. 7D. The $\mathrm{AUC}_{0-24}$ of SP600125 was 31.05 \pm 2.53 and $19.82 \pm 4.57 \mu \mathrm{g} \mathrm{h} / \mathrm{ml}$, and the $\mathrm{C}_{\max }$ was $29.01 \pm 6.64$ and $5.75 \pm 1.06 \mu \mathrm{g} / \mathrm{ml}$ by i.p. and i.g. administration, respectively. Thus, the exposure level of SP600125 in the ANIT-SP-i.p. group was significantly higher compared with that in the ANIT-SP-i.g. group treated with SP600125.

\section{Discussion}

Cholic acid, deoxycholic acid and typical pro-inflammatory cytokines can activate JNK signalling $(21,26)$. The JNK pathway has been reported to regulate the bile acid metabolism $(21,23,27)$. This pathway was activated in cholestatic mice challenged by ANIT and its activation profile was revealed to be correlated with cholestasis and liver injury (26). In the present study, a cholestatic liver injury model was induced by ANIT in mice, and the successful construction of the model was confirmed by serum biochemistry. Obvious loss of cellular boundaries, degenerative changes and marked necrosis were observed in the ANIT group. As anticipated, JNK signalling was activated when cholestasis was induced by ANIT.

The balance among synthesis, uptake and export of bile acids contributes to bile acid homeostasis (25). Bile acids are endogenous ligands for the farnesoid X receptor (FXR), which decreases their synthesis by the SHP-mediated suppression of the genes encoding CYP7A1 and CYP8B1 (27). The activated JNK downregulated the expression of CYP7A1 and CYP8B1 in primary rat hepatocytes (23). In human hepatocytes, FXR induced the expression of fibroblast growth factor 19, a secreted protein that suppresses CYP7A1 through a JNK-dependent pathway (28). In the present study, $C Y P 7 A 1$ and $C Y P 8 B 1$ were downregulated in the cholestatic groups induced by ANIT, while they were not altered in the A-SP-i.p. group, in which JNK signalling was inhibited. Therefore, the downregulation of $C Y P 7 A 1$ and $C Y P 8 B 1$ in the ANIT and A-SP-i.g. groups was the result of both adaptation and JNK-dependent inhibition. $C Y P 7 A 1$ and $C Y P 8 B 1$ levels were not affected in the A-SP-i.p. group, in which cholestasis was also induced and the 
JNK pathway was inhibited. This indicated the dependence of FXR-mediated adaptation on JNK activation, which was in agreement with previously reported findings (29). These data suggested that i.p. administration strongly inhibited JNK signalling and blocked adaptation of synthesis (Fig. 8).

The basolateral uptake transporters, OATP1 transports bile acids from the portal blood into hepatocytes; The basolateral efflux transporter MDR is involved in export of bile acids into the blood and OSTB exports bile acids into canaliculi (25). Bile acids activate FXR, which in turn accelerates bile acid export by inducing BSEP and OST $\beta$ expression, and decreases bile acid uptake by suppressing sodium taurocholate co-transporting polypeptide and OATP expression (30). In the present study, the changes in the bile acid transporters OATPI, OSTB, $M D R 1 A$ and $M D R 2$ were similar among the three groups challenged by ANIT, which were considered as adaptive responses. This indicated that the FXR-mediated transport adaptation was independent of JNK activation, which was different from the synthesis adaptation indicated above.

In the present study, JNK signalling was activated in the ANIT group, but only partially inhibited in the SP-A-i.g. group, as indicated by the increase in p-MKK4, p-JNK and c-Jun in the WB analysis. This serial activation triggered liver injury, which was substantially attenuated when JNK signalling was more markedly inhibited in the SP-A-i.p. group compared with the SP-A-i.g. group. In the present study, SP600125 protected against cholestatic liver injury, as indicated by the regulation of bile acid metabolism, normalization of serum ALT, AST, and ALP levels and histomorphology in the A-SP-i.p. group. Thus, the administration route was found to be crucial for the inhibitory effect of SP600125 on the JNK pathway, which contributed to inhibition of the cholestatic liver injury.

As a JNK inhibitor, SP600125 has been widely used to investigate pathophysiological and pharmacological mechanisms (31). Its concentration used in cellular experiments was usually between 25 and $50 \mu \mathrm{M}(5.5-11 \mu \mathrm{g} / \mathrm{ml})$. In in vivo models of i.g. and i.p. administration, the dose range was $5-30 \mathrm{mg} / \mathrm{kg}(32,33)$. Unfortunately, no studies to date have explored the plasma exposure profile of SP600125. In the present study, the exposure of SP600125 by i.p. injection was significantly higher compared with that by i.g. injection and produced very different exposure profiles. The $\mathrm{AUC}_{0-24}(31.05$ vs. $19.82 \mu \mathrm{g} \mathrm{h} / \mathrm{ml})$ and $\mathrm{C}_{\max }(29.01 \mathrm{vs} .5 .75 \mu \mathrm{g} / \mathrm{ml})$ were statistically significantly different between the two administration routes. The small $\mathrm{T}_{\max }(0.5-1 \mathrm{~h})$ indicated the high potential of SP600125 to be absorbed and distributed. Thus, the in vivo exposure level of SP600125 was close to the concentration of SP600125 used in in vitro models. In the ANIT-SP-i.p. group, the duration of an SP600125 concentration $>5.5 \mu \mathrm{g} / \mathrm{ml}$ was markedly longer compared with that in the ANIT-SP-i.g. group. In cellular models, SP600125 as a competitive inhibitor inhibited the phosphorylation of c-Jun in a dose-dependent manner (33). It may be reasonably inferred that SP600125 at a high concentration binds more JNK and inhibits its signalling. Therefore, the administration route of SP600125 determined its exposure level, and thereby the inhibition of JNK signalling (Fig. 8).

Collectively, the findings of the present study indicated that an effective dose-exposure level was crucial for the inhibitory effect of SP600125 on the JNK pathway, and i.p. administration was more effective compared with i.g. administration. This optimized regimen of SP600125 may lead to an improved design for future in vivo investigations. However, the dose-effect relationship in i.p. administration remains to be investigated for strongest inhibition of JNK signalling without toxic action.

\section{Acknowledgements}

Not applicable.

\section{Funding}

The present study was supported by the Ningbo Natural Science Foundation (grant nos. 2018A610253 and 2018A610384), the Ningbo Public Welfare Project (grant no. 202002N3160), the Zhejiang Public Welfare Technology Research Program (grant no. LGD19H070001), the Zhejiang Provincial Natural Science Foundation of China (grant no. LY20H030001) and the K.C. Wong Magna Fund of Ningbo University.

\section{Availability of data and materials}

The datasets used during the current study are available from the corresponding author on reasonable request.

\section{Authors' contributions}

$\mathrm{XZ}, \mathrm{MD}, \mathrm{JY}$ and AL participated in the research design, conducted experiments, performed data analysis and wrote the manuscript. GX conducted experiments and performed data analysis. LC performed data analysis and contributed new reagents or analytic tools. All authors read and approved the final version of the manuscript to be published.

\section{Ethics approval and consent to participate}

All experiments were approved by the Animal Care and Use Committee of Ningbo University.

\section{Patient consent for publication}

Not applicable.

\section{Competing interests}

Not applicable.

\section{References}

1. Gupta S, Barrett T, Whitmarsh AJ, Cavanagh J, Sluss HK, Derijard B and Davis RJ: Selective interaction of JNK protein kinase isoforms with transcription factors. EMBO J 15: 2760-2770, 1996.

2. Wagner EF and Nebreda AR: Signal integration by JNK and p38 MAPK pathways in cancer development. Nat Rev Cancer 9: 537-549, 2009.

3. Seki E, Brenner DA and Karin M: A liver full of JNK: Signaling in regulation of cell function and disease pathogenesis, and clinical approaches. Gastroenterology 143: 307-320, 2012.

4. Brenner C, Galluzzi L, Kepp O and Kroemer G: Decoding cell death signals in liver inflammation. J Hepatol 59: 583-594, 2013. 
5. Kallunki T, Deng T, Hibi M and Karin M: c-jun Can recruit JNK to phosphorylate dimerization partners via specific docking interactions. Cell 87: 929-939, 1996.

6. Williams AS, Issa R, Leung SY, Nath P, Ferguson GD, Bennett BL, Adcock IM and Chung KF: Attenuation of ozone-induced airway inflammation and hyper-responsiveness by c-Jun NH2 terminal kinase inhibitor SP600125. J Pharmacol Exp Ther 322: 351-359, 2007.

7. Nath P, Eynott P, Leung SY, Adcock IM, Bennett BL and Chung KF: Potential role of c-Jun NH2-terminal kinase in allergic airway inflammation and remodelling: Effects of SP600125. Eur J Pharmacol 506: 273-283, 2005.

8. Assi K, Pillai R, Gomez-Munoz A, Owen D and Salh B: The specific JNK inhibitor SP600125 targets tumour necrosis factor-alpha production and epithelial cell apoptosis in acute murine colitis. Immunology 118: 112-121, 2006.

9. Wang Y, Mei X, Yuan J, Lai X and Xu D: Taurine zinc solid dispersions enhance bile-incubated L02 cell viability and improve liver function by inhibiting ERK 2 and JNK phosphorylation during cholestasis. Toxicology 366-367: 10-19, 2016.

10. Guo C, Wang SL, Xu ST, Wang JG and Song GH: SP600125 reduces lipopolysaccharide-induced apoptosis and restores the early-stage differentiation of osteoblasts inhibited by LPS through the MAPK pathway in MC3T3-E1 cells. Int J Mol Med 35: 1427-1434, 2015.

11. Yan H, Gao Y and Zhang Y: Inhibition of JNK suppresses autophagy and attenuates insulin resistance in a rat model of nonalcoholic fatty liver disease. Mol Med Rep 15: 180-186, 2017.

12. Liu M, Huang G, Wang TT, Sun X and Yu LL: 3-MCPD 1-Palmitate induced tubular cell apoptosis in vivo via JNK/p53 pathways. Toxicol Sci 151: 181-192, 2016.

13. Liu Y, Wang Y, Miao X, Zhou S, Tan Y, Liang G, Zheng Y, Liu Q, Sun J and Cai L: Inhibition of JNK by compound C66 prevents pathological changes of the aorta in STZ-induced diabetes. J Cell Mol Med 18: 1203-1212, 2014

14. Wang Y, Zhou S, Sun W, McClung K, Pan Y, Liang G, Tan Y, Zhao Y, Liu Q, Sun J and Cai L: Inhibition of JNK by novel curcumin analog C66 prevents diabetic cardiomyopathy with a preservation of cardiac metallothionein expression. Am J Physiol Endocrinol Metab 306: E1239-E1247, 2014.

15. Hirschfield GM, Heathcote EJ and Gershwin ME: Pathogenesis of cholestatic liver disease and therapeutic approaches. Gastroenterology 139: 1481-1496, 2010.

16. Hofmann AF: Cholestatic liver disease: Pathophysiology and therapeutic options. Liver (22 Suppl 2): S14-S19, 2002.

17. Huang YH, Chuang JH, Yang YL, Huang CC, Wu CL and Chen CL: Cholestasis downregulate hepcidin expression through inhibiting IL-6-induced phosphorylation of signal transducer and activator of transcription 3 signaling. Lab Invest 89: 1128-1139, 2009.

18. Tan Z, Liu A, Luo M, Yin X, Song D, Dai M, Li P, Chu Z, Zou Z, Ma M, et al: Geniposide inhibits alpha-naphthylisothiocyanate-induced intrahepatic cholestasis: The downregulation of STAT3 and NF[Formula: See text]B signaling plays an important role. Am J Chin Med 44: 721-736, 2016.

19. Miyake JH, Wang SL and Davis RA: Bile acid induction of cytokine expression by macrophages correlates with repression of hepatic cholesterol 7alpha-hydroxylase. J Biol Chem 275: 21805-21808, 2000
20. Higuchi H, Grambihler A, Canbay A, Bronk SF and Gores GJ: Bile acids up-regulate death receptor 5/TRAIL-receptor 2 expression via a c-Jun N-terminal kinase-dependent pathway involving Sp1. J Biol Chem 279: 51-60, 2004.

21. Li D, Zimmerman TL, Thevananther S, Lee HY, Kurie JM and Karpen SJ: Interleukin-1 beta-mediated suppression of RXR: RAR transactivation of the Ntcp promoter is JNK-dependent. J Biol Chem 277: 31416-31422, 2002

22. Li T, Jahan A and Chiang JY: Bile acids and cytokines inhibit the human cholesterol 7 alpha-hydroxylase gene via the JNK/c-jun pathway in human liver cells. Hepatology 43: 1202-1210, 2006.

23. Gupta S, Stravitz RT, Dent P and Hylemon PB: Down-regulation of cholesterol 7alpha-hydroxylase (CYP7A1) gene expression by bile acids in primary rat hepatocytes is mediated by the c-Jun N-terminal kinase pathway. J Biol Chem 276: 15816-15822, 2001.

24. Guo R, Wu H, Yu X, Xu M, Zhang X, Tang L and Wang Z: Simultaneous determination of seven anthraquinone aglycones of crude and processed semen cassiae extracts in rat plasma by UPLC-MS/MS and its application to a comparative pharmacokinetic study. Molecules 22: 1803, 2017.

25. Chiang JY: Bile acid metabolism and signaling. Compr Physiol 3: 1191-1212, 2013

26. Dai M, Yang J, Xie M, Lin J, Luo M, Hua H, Xu G, Lin $H$, Song D, Cheng Y, et al: Inhibition of JNK signalling mediates PPAR $\alpha$-dependent protection against intrahepatic cholestasis by fenofibrate. Br J Pharmacol 174: 3000-3017, 2017.

27. Gonzalez FJ, Jiang C, Xie C and Patterson AD: Intestinal farnesoid $X$ receptor signaling modulates metabolic disease. Dig Dis 35: 178-184, 2017

28. Holt JA, Luo G, Billin AN, Bisi J, McNeill YY, Kozarsky KF Donahee M, Wang DY, Mansfield TA, Kliewer SA, et al: Definition of a novel growth factor-dependent signal cascade for the suppression of bile acid biosynthesis. Genes Dev 17: 1581-1591, 2003.

29. Inagaki T, Choi M, Moschetta A, Peng L, Cummins CL, McDonald JG, Luo G, Jones SA, Goodwin B, Richardson JA, et al: Fibroblast growth factor 15 functions as an enterohepatic signal to regulate bile acid homeostasis. Cell Metab 2: 217-225, 2005.

30. Dawson PA: Role of the intestinal bile acid transporters in bile acid and drug disposition. Handb Exp Pharmacol 169-203, 2011.

31. Wu Q, Wu W, Jacevic V, Franca TCC, Wang X and Kuca K: Selective inhibitors for JNK signalling: A potential targeted therapy in cancer. J Enzyme Inhib Med Chem 35: 574-583, 2020.

32. Hanajiri K, Mitsui H, Maruyama T, Hashimoto N, Sata M and Omata M: Echographic detection of diethylnitrosamine-induced liver tumors in rats and the effect of the intratumoral injection of an inhibitor of c-Jun N-terminal kinase. J Gastroenterol Hepatol 24: 866-871, 2009.

33. Bennett BL, Sasaki DT, Murray BW, O'Leary EC, Sakata ST, Xu W, Leisten JC, Motiwala A, Pierce S, Satoh Y, et al: SP600125, an anthrapyrazolone inhibitor of Jun N-terminal kinase. Proc Natl Acad Sci USA 98: 13681-13686, 2001. 